\title{
Welchen Platz nimmt Stensens anatomische Forschung in Lorenz Heisters Chirurgie und Anatomie ein?
}

\author{
Von Adolf Faller
}

\section{Einleitung}

Die Lebensdaten von Niels Stensen (1638-1686) und Lorenz Heister (1683-1758) überschneiden sich kaum. Als Stensen im Alter von nur 48 Jahren starb, war Heister noch ein Kind. In Lebenslauf und Charakter sind beide grundverschieden. Was veranlaßt uns, Stensen, den Biologen mit Heister, dem großen Erneuerer der Chirurgie im deutschen Sprachraum zu vergleichen? Lichtenthaeler hat 1974 in seiner «Geschichte der Medizin» Heister zusammen mit Morgagni und Haller zu den großen Enzyklopädisten des 18. Jahrhunderts gerechnet. Auf dem Hintergrund der Chirurgie und der Anatomie kann uns Heister zeigen, was an Stensens anatomisch-biologischen Entdeckungen von den ihm zeitlich Nahestehenden allgemein anerkannt und besonders geschätzt wurde.

\section{Niels Stensen 1638--1686}

Niels Stensen wurde als Sohn eines Goldschmiedes in Kopenhagen geboren. Er studierte in Kopenhagen, Amsterdam und Leiden nur deshalb Medizin, weil das damals der einzige Weg war, sich einigermaßen systematisch in «Biologie» auszubilden. Stensen hat sich nie als praktischer Arzt niedergelassen und war auch niemals als akademischer Lehrer tätig. Ihn interessierte die eben in Entstehung begriffene «Biologie». Die «neuen» Ideen waren vor allem der methodische Zweifel von Descartes und die strenge Empirie der Forschung im naturwissenschaftlichen Bereich im Sinne von Galilei. Diese Ideen waren weniger an Universitäten beheimatet. Vielmehr interessierten sich dafür begabte Forscher um einzelne besonders berühmte Mäzene oder Wissenschaftler, wie etwa Melchisédech Thévenot in Paris oder Kardinal Leopoldo de Medicis, der Begründer der Accademia del Cimento in Florenz und Beschützer Galileis. Die notwendigen Mittel wurden von den regierenden Fürsten zur Verfügung gestellt, die auch für die Drucklegung der 
entsprechenden Werke aufkamen. Stensen war kein systematischer Forscher auf einem genau bestimmten Gebiet. Ihn interessierte alles, was neue Erkenntnis vom Lebenden versprach. Ob es sich dabei um vergleichende Anatomie, systematische Anatomie, Physiologie oder Embryologie handelte, ob er ganz neue Gebiete der Wissenschaft erschloß, wie etwa Hirnanatomie, Paläontologie, Geologie und Kristallographie, war für ihn unerheblich. Entscheidend war allein der Drang nach neuer und vertiefter Erkenntnis des «Lebendigen». Der jeweilige Gegenstand seiner Forschung wurde ihm häufig durch äußere Umstände zugeteilt. Das gilt nicht nur für seine naturwissenschaftlichen Untersuchungen, sondern auch für seine spätern philosophisch-theologischen Arbeiten, die zu seiner Konversion führten und ihn Priester und Bischof werden ließen. Schließlich kannte er nur eine einzige Sorge, Gott über alles zu dienen und die Mitmenschen zu Gott zu führen. Er starb arm und verlassen im Rufe der Heiligkeit 1686 auf der Missionsstation Schwerin.

Lorenz Heister 1683-1758

Lorenz Heister wurde als Sohn eines Gastwirtes in Frankfurt a. M. geboren. Er studierte Medizin in Gießen, Wetzlar, Leiden sowie Amsterdam und promovierte in Harderwyk. Als Militärarzt und Kriegschirurge erwarb er sich eine große praktische Erfahrung. 1710 wurde er Professor der Anatomie und der Chirurgie an der Universität Altorf. 1719 erhielt er einen Ruf nach Helmstädt, wo er bis zu seinem Tode als Chirurge tätig war. 1730 vertauschte er die Professur für Anatomie mit derjenigen für Botanik und theoretische Medizin. Nach dem Tode seines Kollegen Meibom übernahm er 1740 die Professur für praktische Medizin, welche er bis zu seinem Tode inne hatte. Er starb mit 75 Jahren, erreichte somit ein für die damalige Zeit hohes Alter. Während 35 Jahren lehrte er an der Universität Helmstädt. Er war ein ausgezeichneter praktischer Arzt mit einer ungewöhnlichen Erfahrung in Chirurgie. Er war ungemein belesen: 12000 Bände standen in seiner Privatbibliothek. Heinrich Haeser charakterisiert ihn (1881) zutreffend: «Derselbe hat die Chirurgie mit wesentlichen Fortschritten nicht bereichert, wohl aber in seinem wohlgeordneten, zu seiner Zeit hochangesehenen Lehrbuch alles zusammengefaßt, was bis dahin Bleibendes gewonnen worden war». Von Heister zitiert zu werden, galt als Zeichen besonderer fachlicher Zuverlässigkeit und Bedeutung. Pagel sagt im Kapitel «Die 


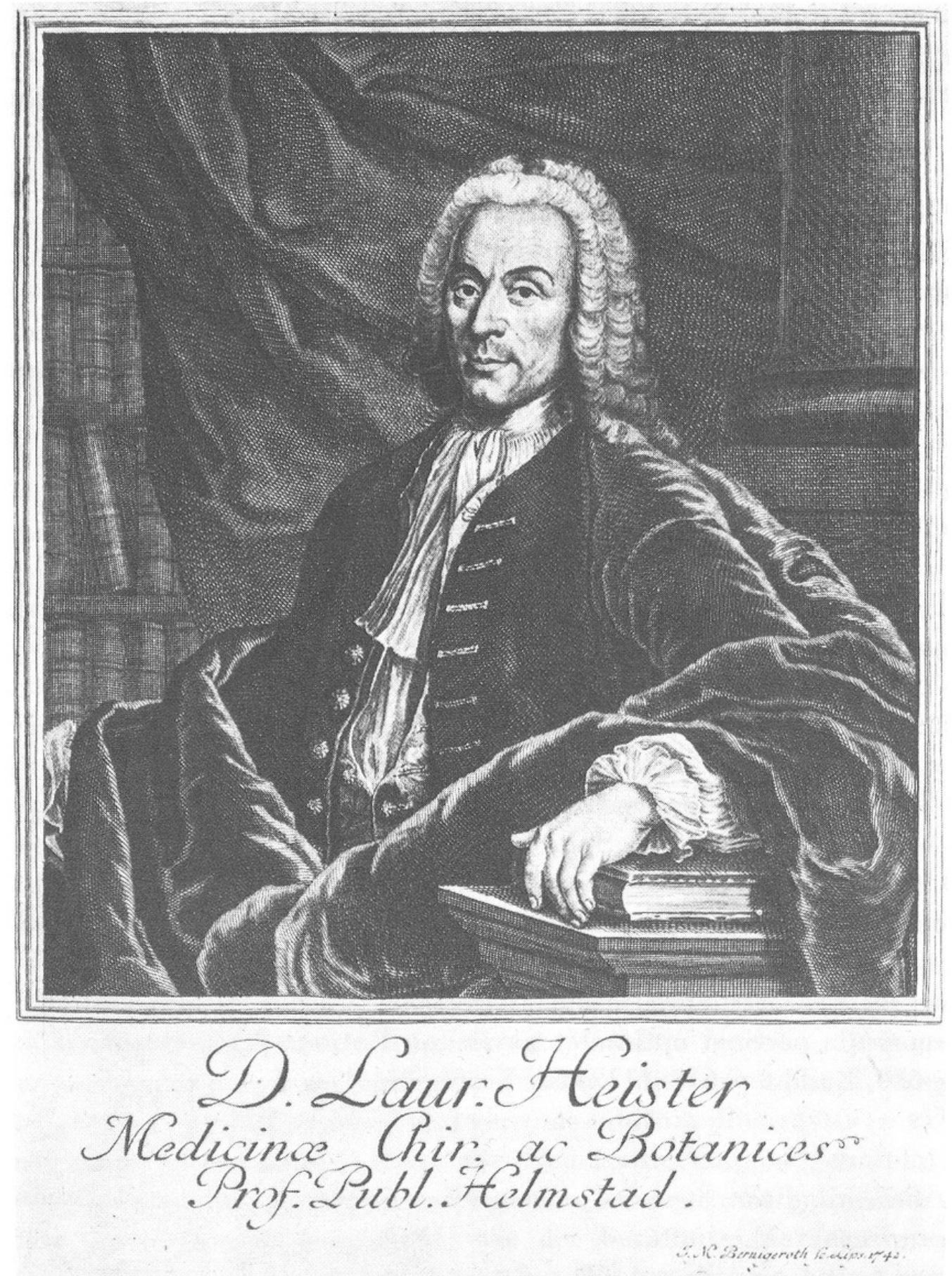

Abb.1. Der 59jährige Lorenz Heister auf der Höhe seines Ruhmes in Helmstädt. In Perücke und akademischer Toga sitzt Heister in seiner geliebten Bibliothek. Der Kupferstich wurde 1742 von J. M. Bernigeroht in Leipzig hergestellt. 
Chirurgie im 18. Jahrhundert» seines Lehrbuches (1898): «Heister hat das Verdienst, die Chirurgie in Deutschland wieder zu Ehren gebracht zu haben. Verdankt ihm auch diese Wissenschaft keine eigentliche Bereicherung, so hat er doch durch ein Lehrbuch, das lange Zeit (bis zum Erscheinen desjenigen von August Gottlob Richter) in großem Ansehen stand, sich um den Unterricht direkt und indirekt sehr verdient gemacht ... Übrigens war Heister auch ein tüchtiger Anatome und Verfasser eines beliebten Compendium Altorf 1717.» Haberling in Hirsch (1962) rechnet ihn «zu den vielseitigsten Männern unserer Wissenschaft. Außer der Anatomie, Chirurgie, Augenheilkunde und Botanik, in welchen er Treffliches leistete, war er auch vieler Sprachen und auch der Naturwissenschaften in seltenem Grade kundig. Im Interesse des Studiums und der Darstellung erlernte er auch das Glasschleifen und Kupferstechen. Heister ist kein Bahnbrecher. Aber er hat durch Prüfung und Verbreitung der von anderen gefundenen Tatsachen die Wissenschaft gefördert ... Seine Chirurgie verdankt ihren großen Erfolg dem Umstande, daß Heister die besten Quellen benutzte und seinen Lehren überall die anatomische Grundlage zu geben wußte». Die Genialität, die wir an Stensen so sehr bewundern, fehlt Heister. An deren Stelle treten Erfahrung und große Belesenheit. Was in seinen weit verbreiteten Lehrbüchern der Anatomie und der Chirurgie Aufnahme fand, durfte zum gesicherten Stand der Medizin gerechnet werden. Aus diesem Grund haben wir uns die Mühe genommen, den Spuren Stensens bei Heister nachzugehen. Zur Lektüre standen mir die beiden Bände «Laurentii Heisteri Compendium Anatomicum totam Rem anatomicam complectens» der lateinischen Ausgabe Amsterdam 1771 und die zweibändigen 1740 in Venedig erschienenen «Laurentii Heisteri Institutiones Chirurgicae, in quibus quicquid ad Rem chirurgicam pertinet optima et novissima Ratione pertractatur» (Band 1 p. 1-640, Band 2 p. 643-1112) zur Verfügung. *

Die Bedeutung von Stensens anatomischer Forschung für die «Institutiones chirurgicae»

Mit sicherer Hand hat Heister zwei anatomische Entdeckungen Stensens in ihrer Bedeutung für die chirurgische Praxis herausgegriffen: den Ausführgang der Ohrspeicheldrüse und die ableitenden Tränenwege:

* Die Seitenzahlen meiner Heister-Zitate beruhen auf diesen Ausgaben. 
«Stenonianus ductus vulneratus» (p. 122): «Genarum sive malarum vulnera eodem prorsus modo \& cum eadem circumspectione tractari debent, atque ea, quae labris inflicta sunt (Vid. No 9). Sie quis autem ex Stenonianis illis qui per transversam genam e glandula parotide procedunt ductus salivalis praecisus est, sane vulnus ejusmodi propter salivam continuo, atque inter masticandum praesertim, quasi per fistulam aliquam inde propullulantem (a) glutinari vix ac ne vix quidem poterit, quam perforato intus ductu, saliva omnis in os effundatur. Namque cum demum curari feliciter externa genarum vulnera, Cheseldeno, anglo chirurgo, authore (b) consueverunt» - «Verletzungen der W angen oder der Backen sind wie auch diejenigen der Lippen (s. No 9) mit derselben Vorsicht zu behandeln. Wenn aber einer der Speichelgänge Stensens verletzt ist, die aus der Parotisdrüse quer über die $W$ ange laufen, so kann eine solche $W$ unde wegen des ständigen Speichelflusses besonders während des Kauens nur durch eine Art Fistelbildung nach innen (a), wodurch aller Speichel in die Mundhöhle entleert wird, verheilen. Nur so pflegen nach dem englischen Chirurgen Cheselden (b) solche äußere Wunden sich gut zu schließen». a) ist ein Hinweis auf Fabricius ab Aquapendente, Hildanus und Nuck, b) verweist auf das englisch geschriebene Anatomiebuch von Cheselden. Heister benützt die topographische Anatomie zur Erklärung des chirurgischen Verhaltens. Die Hinweise a und $b$ zeigen, daß er das einschlägige Schrifttum genau gekannt hat.

«De Epiphora sive oculo lacrymante» (p.507): Die Anmerkung (a) zum Kapitel 53: «Zum Tränentreuffeln» gibt Heister Gelegenheit, den ganzen Fragenkomplex der abführenden Tränenwege aufzurollen und Stensen den ihm gebührenden Platz einzuräumen: «Has vias lacrymales haud pauci pro novis inventis habuerunt, verum ea iam Galeno, Vegetio, Berengario, Fallopio, Carcano, Stenoni, aliisque perspectas fuisse, ostendit cl. Morgagnus in Advers. anatom. 1 und 6 qui vero eas multum illustravit». - «Diese Tränenwege haben nicht wenige für Neu-Entdeckungen gehalten: Sie wurden jedoch schon von Galen, Vegetius, Berengar, Fallopio, Carcano, Stensen und anderen festgestellt, wie der berümte Morgagni in den Adversaria anatomica 1 und 6 gezeigt hat. Er hat viel zu ihrer Kenntnis beigetragen». Für Stensen waren die Tränenwege vor allem ein anatomisches Argument gegen Descartes. Wer in einer solch einfachen anatomischen Frage geirrt hat, dem können auch bei den viel schwierigeren Fragen, welche die Seele des Menschen und Gott betreffen, ein Irrtum unterlaufen sein. Stensens Kritik des Cartesianismus nimmt ihren Ausgang vom anatomi- 
schen Befund. Den stärksten Ausdruck dieser vom Anatomischen ausgehenden Zweifel haben ihren Niederschlag in Stensens «Discours sur l'anatomie du cerveau» gefunden, worin er gezeigt hat, daß das Corpus pineale sich in seiner Lage nicht bewegen läßt. Mit Stensens anatomischen Bedenken beginnt eine erste Phase der Kritik am Cartesianismus.

Welchen Platz nehmen Stensens anatomische Untersuchungen in "Laurentii Heisteri Compendium Anatomicum» ein?

Heisters «Compendium Anatomicum» umfaßt zwei Bücher und erschien 1717 erstmals in Altdorf bei Nürnberg. Es erlebte 12 Auflagen und zahlreiche Übersetzungen. Stensen ist mit Ruysch einer der am häufigsten zitierten Anatomen. $15 \mathrm{mal}$ verweist Heister auf «Stenonis», was wir wohl mit Sohn des Sten zu übersetzen haben. Fr rechnet ihn «ex recentioribus», das heißt zu den neueren Anatomen, deren Gruppe von Harvey bis Havers reicht. Diese schließen sich an die «primarii \& quasi principes» an und werden von der Gruppe der zeitgenössischen Anatomen, die als «ex recentissimis» angesprochen werden, gefolgt. So belesen Heister auch war, es blieb für ihn doch die selber gemachte eigene Erfahrung, als deren Mittel er Präparation, Mikroskop und Injektion auffüht, die Hauptsache. Entsprechend gruppiert er in der Einleitung: «1. Diligens observatio, quando anatomici exercitati cadavera incidunt; 2 . Imitatio, tam in bestiis quam in hominibus; 3. Bonorum scriptorum lectio» - «1. Genaue Beobachtung, wenn FachAnatomen Leichen präparieren; 2. Eigene Arbeit, die solchen Vorbildern folgt, sowohl an tierischen wie auch an menschlichen Körpern; 3. Das Lesen guter anatomischer Schriftsteller» (S.2). Stensens anatomische Untersuchungen werden besonders bei den Drüsen und ihren Ausführgängen, bei einzelnen Beobachtungen aus der Muskellehre und bei der Erwähnung der Funktion der Eierstöcke herangezogen. Erstaunlicherweise findet sich kein einziger Hinweis auf Stensens «Discours sur l'anatomie du cerveau». Entweder hat Heister diese Arbeit nicht gekannt, was bei seiner Belesenheit und seiner Beherrschung der französischen Sprache erstaunen dürfte oder er hat ihre positive Bedeutung für die Hirnanatomie unterschätzt.

Drüsenlehre und Lehre von deren Ausführgängen: Hier handelt es sich um die Tränendrüsen und die Tränenwege, die Glandula parotis, die Glandula sublingualis, die Schweißdrüsen der Haut und den Canalis nasopalatinus. Bei Stensen konzentriert sich das Interesse auf eine Auseinandersetzung mit 
der cartesianischen Anatomie. In der Fußnote f (p. 142) erwähnt Heister Stensen bei der Tränendrüse: «Glandula lacrymalis, in orbita, supra angulum minorem sita, cum ductulis suis excretoriis sub palpebra superiori (f) emergentibus, facilius in bobus quam homine demonstrandis» - «Die Tränendrüse liegt in der Augenhöhle über deren kleinerem Winkel. Ihre Ausführgänge treten unter dem Oberlid aus. Sie sind bei Rindern leichter darzustellen als beim Menschen». Als Referenz gibt er «Steno de glandul. oculor.» an. In Nota $49 * *$ ad pag. 142: «Viae lacrymarum ex oculo ad nares non sunt recentiorum Anatomicorum inventa, ut multi putarunt, postquam ab aliquo tempore quamplurimis neglectae aut ignotae fuerunt; neque eorum cognitio ad usum medicum \& cum primis chirurgicum applicata fuit, donec tandem hoc seculo Anellus, forte ex lectioni Advers. anat. I n. 22, \& post Anellum ego, in obstructione canalis oculo lacrymante \& fistulsa lacrymali curandis, haud infelicter in praxin chirurgicam introduxerimus»«In der Anmerkung 49** zu S.142: Die Tränenwege vom Auge zu den Nasenhöhlen sind keineswegs eine Beobachtung der neueren Anatomen, wie viele geglaubt haben, nachdem sie eine Zeitlang von vielen vernachlässigt und nicht mehr bekannt waren. Ihre Kenntnis wurde weder in der Medizin noch in der Chirurgie angewendet, bis in diesem Jahrhundert, vielleicht aufgrund der Lektüre der Advers anat. 1 und 22 Annellus und nach ihm ich bei Verstopfung des Tränennasenkanals, beim tränenden Auge und bei der Tränenfistel mit Erfolg in der praktischen Chirurgie sie wieder einführten.» Beschrieben haben dieselben Galen, Vegetius, Berengarius, Fallopio, Fabricius ab Aquapendente, Stensen, Morgagni, Meibom und Heister. Für Stensen verweist er auf die «Obs. anat. de Glandulis p. $91 »$.

In Band 2 hat Heister die Parotisdrüsen recht gut geschildert: «insignes glandulae, utrinque una, inter aures et maxillae inferioris angulum sita, saepe super magnam masseteris partem se extendens: ex qua notabilis ductus, tres quasi transversos digitos longus, calami straminei crassitie, multis radicibus prodit, qui a Stenone An. 1660 inventus, (a) Ductus salivalis Stenonianus, sive superior, appellatur» (p. 145) - «Es sind beachtliche Drüsen auf jeder Seite eine, zwischen den Ohren und dem Unterkieferwinkel gelegen und bedecken einen großen Teil des Massetermuskels. Daraus geht ein beachtlicher Gang von etwa 3 Querfingern Länge und der Dicke eines Strohhalmes mit vielen Wurzeln hervor. Er wurde von Stensen 1660 entdeckt und wird deshalb Stensengang oder oberer Speichelgang genannt». In der Nota $55^{*}$ p. 106 des 2. Bandes greift Heister erneut auf Stensens Untersuchungen der Speicheldrüsen zurück: «Glandulas sublinguales vas- 
cula excretoria utrinque ad linguae latera obtinere brevissima \& angustissima, sibi invicem parallela, vixque conspicua, nisi premantur, Steno prius in brutis notavit atque delineavit (b); deinde Stenonis verbis, ejus tamen nomine suppresso, ea quoque indicavit Verhejenus (c)»-«Die Unterzungenspeicheldrüsen haben zu beiden Seiten der Zunge sehr kurze und sehr enge, einander parallel laufende Ausführgänge, die man kaum sieht, wenn man sie nicht ausdrückt, Stensen hatte sie bei Tieren beobachtet und auch gezeichnet; dann hat sie Verheyen mit Stensens Worten, aber ohne dessen Namen anzugeben, erwähnt». (b) ist ein Hinweis auf «Observ. anatom. de Glandul. oris», $\$ 20$ fig. 3., (c) ein solcher auf «Anatome Tract. IV. cap. XX».

Von mehr Interesse ist die Erwähnung Stensens auf p. 70 des 1. Buches im Kapitel «De cute». Sie betrifft die Schweißdrüsen: «Glandulae cutaneae miliares copiosissimae, a plerisque recentioribus, post Stenonem \& Malpighium, in cute ubique statuuntur, pro secretione materiae exspirandae; quae tamen vix vel saltem paucissimae, demonstrari possunt, earumque attributa functio a solis in cute arteriolis fieri potest» - «Die körnchenartigen Hautdrüsen werden nach Stensen und Malpighi überall in der Haut gefunden. Sie dienen der Absonderung des Schweißes, lassen sich aber nur selten und in geringer Anzahl präparieren. Thre Funktion ist an die kleinen Arterien der Haut gebunden». Im 2. Band im Kapitel «De glandulis speciatim» p. 35/36 werden erneut die Schweißdrüsen erwähnt: «Verhejenus (Anat. cap. de cute cap. 35) inquit Stenonem observasse, singulis poris suam glandulam subjacere, ex qua vasculum sudoriferum, in exterioris cutis superficie desinens, exurgeret» - «Verhejen sagt, nach Stensen soll unter jeder Hautpore eine Drüse liegen, aus welcher zur Hautoberfläche führend ein Ausführungsgang hervorgehe». Heister lehnt das mit der Begründung ab, daß es soviele Hautdrüsen gar nicht geben könne. Er hält das Axiom (p.36) «ubicunque secretio ibi glandula» für irrig, von welchem Stensen und andere ausgegangen waren. Heister verwirft hier eine beobachtbare Tatsache, nur weil sie ihm nicht wahrscheinlich vorgekommen ist. Jedermann kann sich davon überzeugen, daß bei ausbrechendem Schweiß auf den Leisten der Haut von Handfläche und Fußsohle eine Unzahl kleinster glänzender Pünktchen auftreten. In der Nota $3^{*}$ p. 52 kommt er darauf zurück: «... ex quibus vero minimum id evictum esse credo, glandulas veras culaneas tanta copia non dari, ut cuivis foraminulo cutis insensibili, sive unicuique poro, una subjecta sit: sicuti Steno, \& multi alii post eum, crediderunt atque docuerunt» - «Aus all dem scheint mindestens das klar hervorzugehen, daß es eigentliche Hautdrüsen in solcher Zahl nicht gibt, daß jedem kleinsten 
Löchlein, jeder Pore eine darunterliegende Drüse entspräche, wie Stensen und viele andere nach ihm geglaubt und gelehrt haben.»

Auch der "Canalis incisivus» der heutigen "Nomina anatomica» wurde mit Sekretionserscheinungen in Zusammenhang gebracht. Die Anmerkung (a) auf p. 35 des 1. Buches lautet «Plerique anatomici recentiores liquidum per hunc canalem ex naso in os influere sustinent, atque hanc ob causam eum Stenonis transitum a naribus ad palatum vocant, qui etiam in capite osseo satis apertus et et amplus apparet» - «Die meisten neueren Anatomen vertreten deshalb die Ansicht, es fließe Flüssigkeit aus den Nasenhöhlen in den Mund und sprechen deshalb von der «Stensenverbindung zwischen Nasenhöhlen und Gaumen». Sie erscheint am knöchernen Schädel offen und genügend weit. Tatsächlich treten Äste des N. nasopalatinus auf diesem Wege durch zur Versorgung des Zahnfleisches im Zwischenkiefergebiet.

Einzelne Beobachtungen aus der Muskellehre: Die Anmerkung (f) verweist auf die Lehre von den Zungenmuskeln bei Malpighi, Fracassatus und Steno. Heister schreibt auf p. 150 des 1. Buches von den Zungenmuskeln: «Quorum, praeter eos quos os hyoides habet, quatuor sunt paria, de quibus in Myologia: super quos vero multae adhuc aliae musculosae inexetricabiles occurrunt fibrae; (f), quibus linguae contrahitur, expanditur, protrahitur, retrahitur, ceterique omnes mirabiles linguae motus in loquela \& manducatione perficiuntur» - «außer der Zungenbeinmuskulatur kommen vier einander ähnliche vor, davon mehr in der Myologie. Darüber liegen andere, schwer zu analysierende Muskelfasern, mit deren Hilfe die Zunge zusammengezogen wird, erschlafft, nach vorne oder nach hinten gezogen wird. Es werden damit alle staunenswerte Bewegungen der Zunge beim Sprechen und Kauen hervorgebracht.» Stensen hat sich dahin geäußert, daß er den Plan habe, eine vergleichende Muskellehre zu schreiben. Leider ist dieser Vorsatz aus Zeitmangel nie zur Ausführung gekommen. Bei den Atemmuskeln erwähnt Heister auf p. 188 besonders den «Levator costarum Stenonis», fügt aber in der Fußnote (a) hinzu, daß schon Casserius ihn gekannt habe. Steno, Borelli und Lower haben nach Heister neben den einfachen Muskeln auch zusammengesetzte «compositi» gekannt. Auf p. 172 gibt Heister auch einen Hinweis auf den Stensenversuch am Hund, wobei die Durchblutung des Muskels behindert wird: «Sicuti vero musculus, nervo ejus dissecto, velliato, agere amplius nequit; ita eum quoque agere non posse, ligata vel dissecta arteria, Stenonis, aliorumque in canibus experimentis \& industria compertum est» - «Ein Muskel kann nicht mehr arbeiten, wenn sein Nerv durchschnitten oder unterbunden wird; seine Tätigkeit geht aber auch 
verloren, wenn dessen Arterie unterbunden oder durchgeschnitten wird. Stensen und andere haben das in Experimenten an Iunden mit Geschick nachgewiesen» (p.172 des 1. Buches).

Der Streit um die Funktion der Eierstöcke: Zur Zeit Stensens betrachtete man häufig die Eierstöcke als Rudimente von Hodenanlagen bei der Frau, «testes muliebres». Die Eizelle als weibliche Keimzelle war weder Stensen noch Heister bekannt. Die entsprechenden Kenntnisse der mikroskopischen Anatomie fehlten noch völlig. Erst die Untersuchungen von Carl Ernst von Baer erbrachten 1827 den Nachweis der Eizelle im Epithel des Follikelbläschens. «Ovulum» oder «Ovum» sind zunächst Begriffe der makroskopischen Anatomie und bedeuten nicht «Eizelle» im heutigen Sinn, sondern sind Synonyme für Ei-Bläschen oder Bläschenfollikel. Wenn van Horne, de Graaf und Kerkring sowie Stensen von «Vesiculae rotundae» im Eierstock sprechen, so sind stets Bläschenfollikel damit gemeint (vergl. p. 105): «Vesicule rotundae plures, pauciores, pro aetatis \& temperamenti diversitate, humore albo ovi simili, repletae; qui humor ebulliendo consistentiam, colorem \& gustum quoque albi ovi simili adipiscitur; hine ob analogiam cum ovis a Stenone primum dicta sunt ovula, quorum maxima pisum vix aequant. Quandoque 10, 15, 20, imo plures in uno ovario vix unum vel alterum observari potest: creduntur prima foetus rudimenta continere»«Die runden Bläschen sind bald zahlreich, bald selten je nach Alter und Veranlagung. Sie sind von einer dem Hühnereiweiß gleichenden Flüssigkeit gefüllt. Diese Flüssigkeit erhält beim Kochen Konsistenz, Farbe und Geschmack von Hühnereiweiß. Wegen dieser Ähnlichkeit mit dem Hühnerei wurden sie erstmals von Stensen kleine Eier genannt. Ihre Größe kann die einer Erbse erreichen. Gelegentlich werden 10, 15, 20, ja sogar mehr in einem Eierstock beobachtet. Man nimmt an, daß sie die Anlage der Frucht enthalten.» Der Hinweis (a) beruft sich auf Stensens «Diss. pisc. ex can. gen.». Daß Heister auch diese Annahme erwähnt, ist ihm hoch anzurechnen. Er ist damit der Wahrheit sehr nahe gekommen und hat damit eine wichtige Brücke zu den spätern Ergebnissen der mikroskopischen Anatomie geschlagen. Die Begriffe Hühnerei, Bläschenfollikel und Eizelle beginnen sich allmählich voneinander abzugrenzen. Heister hat dazu wesentlich beigetragen.

Die Neuroanatomie: Erstaunlicherweise findet man in Heisters Chirurgie und auch in seiner Anatomie keinerlei Hinweis auf Stensens «Discours sur l'anatomie du cerveau», welchen dieser 1665 in Paris gehalten hat, der aber erst 1669 im Druck erschienen ist. Entweder hat Heister diese Publikation 
gar nicht gekannt, was bei seiner Belesenheit und seinen Sprachkenntnissen verwundern dürfte, oder er hat deren Bedeutung für seine Zeit unterschätzt. Die Programmrede für die zukünftige Hirnforschung schien ihm für die damalige Hirnanatomie wenig zu bieten.

\section{Zusammenfassung}

Die Kommentare in den Lehrbüchern der Chirurgie und auch der Anatomie Heisters geben eine gute Vorstellung davon, wie Stensens anatomische Untersuchungen von seinen Fachkollegen eingeschätzt wurden. Am meisten Interesse haben Stensens Ergebnisse auf dem Gebiet der Drüsen- und Muskelforschung sowie in der Erkenntnis von der Funktion der Eierstöcke gefunden. Heister kennt den «Stensen-Versuch〉, der die Bedeutung der Blutzirkulation für die Muskelfunktion hervorhebt. Ein Hinweis auf Stensens Programmrede zur Hirnforschung fehlt bei Heister. Sie schien ihm zuwenig Aktuelles für die damalige Hirnanatomie zu bieten.

\section{Benütztes Schriftum}

Bierbaum, M. $\dagger$ und Faller, A.: Niels Stensen, Anatom, Geologe und Bischof. 203 S. Aschendorff, Münster-Westf. 1959. Faller, A.: Wertschätzung von Stensens «Discours sur l'anatomie du cerveau» im Verlaufe von drei Jahrhunderten. Veröffentl. Schweiz. Ges. Geschichte d. Medizin u. d. Naturw. Nr.35 96 S. Sauerländer Aarau-Frankfurt a. M. 1981. Faller, A.: Elemente einer Wissenschaftslehre und einer Wissenschaftskritik in den Schriften von Niels Stensen 1638-1686. Gesnerus 37 (1980) 169-188. Haeser, H.: Lehrbuch d. Geschichte der Medicin u. der epidemischen Krankheiten. 3. Aufl. Bd.2: Geschichte d. Medicin in der neueren Zeit. 1116 S. Fischer, Jena, 1881. Heisterus, L.: Institutiones Chirurgicae, in quibus quicquid Ad Rem Chirurgicam Pertinet, Optima et Novissima Ratione Pertractatur Tom. I et II. 1110 p. Venetiis 1740. Heisterus, L.: Compendium Anatomicum, Totam Rem Anatomicam brevissime complectens. Tom.I, 207 p. Tom.II, 190 p. Amstelodami 1771. Hirsch, A.: Biogr. Lexikon der hervorragenden Ärzte aller Zeiten und aller Völker. 3. Aufl. Bd.3, 873 S. Urban \& Schwarzenberg, München-Berlin 1962; Bd.5, 1058 S. Urban \& Schwarzenberg, München-Berlin 1962. Kruse, H.: Die Augenheilkunde bei Lorenz Heister. Med. Diss. Jena, 66 S. Studentenhaus München 1929. Lichtenthaeler, Ch.: Geschichte der Medizin in 2 Bden. 736 S. Deutscher Ärzteverlag, Köln-Lövenich 1974. Maar, V.: Nicolai Stenonis Opera Philosophica. Vol. I et II. V.Tryde Copenhagen 1910264 resp. 365 p. Pagel, J.: Einführung in die Geschichte der Medicin. 25 Akad. Vorl., 574 S. Karger, Berlin 1898. Puschmann, Th.: Handbuch der Geschichte der Medizin von Neuburger, M. und Pagel, J., Bd.2, 960 S. Bd. 3, $1128 \mathrm{~S}$. 


\section{Summary}

A reading of the text books by Lorenz Heister on anatomy and surgery gives a good idea of in how high esteem were held Stensen's anatomy of glands and muscles as well as of ovaries and their function. Heister was also aware of "Stensen's experiment", without however, making any mention of Stensen's programmatic "Discours" en cerebral research, which, at that time, was of too little practical interest, pointing, moreover, to the far future.

Prof. Dr.med. Adolf Faller

10 , chemin St-Marc

CH-1700 Fribourg 